\title{
Efecto del tipo de prueba de evaluación en la memoria y valoración de marcas publicitarias
}

\section{Effect of the type of memory test on the evaluation of brands}

\author{
Beatriz Martín-Luengo ${ }^{1}$, Karlos Luna ${ }^{2}$ y Malen Migueles ${ }^{1}$ \\ ${ }^{1}$ Facultad de Psicología, Universidad del País Vasco, España. \\ ${ }^{2}$ School of Psychology, University of Minho, Portugal.
}

Disponible online 31 de diciembre de 2012

\begin{abstract}
Una de las maneras más frecuentes de medir la efectividad de un anuncio publicitario es mediante el uso de pruebas de memoria. Sin embargo, cada prueba de evaluación aporta un tipo de información diferente por lo que es importante saber cuál es la más conveniente. El objetivo principal de esta investigación ha sido examinar el efecto que tiene el tipo de prueba de memoria tanto en el recuerdo de marcas publicitarias como en el interés subjetivo que estas generan. Un grupo de participantes escribieron marcas de productos que recordaran haber visto anunciados (prueba de recuerdo libre) y valoraron el interés por cada marca. Después recibieron un listado de 27 categorías y escribieron marcas de productos asociadas a ellas (prueba de recuerdo con clave) y también valoraron su interés. Los resultados muestran que con la prueba de recuerdo con clave se generó un mayor número de marcas, pero que las generadas en la tarea de recuerdo libre fueron evaluadas con mayores niveles de interés. Estos resultados muestran la importancia de escoger una tarea de memoria adecuada para medir el efecto de la publicidad.
\end{abstract}

Palabras clave: Recuerdo Libre; Recuerdo con Clave; Interés; Publicidad; Marcas.

\begin{abstract}
Memory tests are frequently used to measure the effectiveness of an advertisement. However, as different tests provide different kinds of information it is important to know which type of test is the most appropriate. The main objective of this investigation was to study the effect of different memory tests on the number of brands recalled and the level of interest generated by them. A group of participants wrote down all the brands of any kind of product that they remembered having seen advertised (free recall test) and rated their interest in each brand. Subsequently, the participants were given a list of 27 categories and wrote down the brands they associated with the categories (cued recall test) and also rated their level of interest. The results showed that although the participants generated more brand names with the cued recall test, the brand names mentioned in the free recall test generated greater interest. These results highlight the importance of choosing the correct memory test to measure the effect of publicity.
\end{abstract}

Key words: Free Recall; Cued Recall; Interest; Publicity; Brands.

Correspondencia: Beatriz Martín-Luengo. Dpto. Procesos Psicológicos Básicos y su Desarrollo, Facultad de Psicología, Universidad del País Vasco, UPV-EHU. Avda. Tolosa, 70, 20018. Donostia-San Sebastián, Gipuzkoa, España. Tlfs.: (+34) 9430180 65. Fax: 943.01 56 70. E-mail: beatriz.martin.luengo@gmail.com. E-mails de los otros autores: Karlos Luna: karlos.luna@psi.uminho.pt. Malen Migueles: malen.migueles@ehu.es Este trabajo ha sido subvencionado por una beca FPU del Gobierno Vasco concedida a la primera autora. 
"Si no te recuerdan no importa lo bueno que seas". Además de ser el eslogan de la última campaña de promoción de espacios publicitarios de Comfersa (Comercial del Ferrocarril, S.A.), también identifica el objetivo prioritario de la publicidad: que los potenciales compradores recuerden la marca anunciada (L. Sánchez, Megías y Rodríguez, 2004). Sin embargo, la información que seamos capaces de recordar depende del tipo de prueba de memoria y de cómo estén planteadas las preguntas (Goldsmith, Koriat y Weinberg-Eliezer, 2002; Luna, Higham y Martín-Luengo, 2011). El objetivo principal de este trabajo es examinar si el tipo de prueba de memoria utilizada determina el recuerdo de productos publicitarios.

Dentro de los estudios de publicidad, los índices más habituales para medir la efectividad de los anuncios son medidas de memoria, ya sean tareas de recuerdo libre, de reconocimiento o de recuerdo con clave (Fennis y Stroebe, 2010; Norris y Colman, 1996; Norris, Colman y Alexio, 2001, 2003). Por ejemplo, Norris y cols. (2003) evaluaron la memoria con las tres pruebas de memoria. Tras presentar los anuncios, primero midieron recuerdo libre (enumerar todas las marcas presentes en los anuncios), después recuerdo con clave a partir de categorías de productos, y finalmente proporcionaron una prueba de reconocimiento. Las tres medidas son pruebas validas de memoria, sin embargo, la información que nos proporciona cada una de ellas es diferente (Richardson-Klavehn y Bjork, 1988). La prueba de recuerdo libre nos informa sobre lo que un participante es capaz de recordar de manera espontánea. La prueba de recuerdo con clave y el nombre de la categoría nos facilita la tarea de búsqueda de información, por lo que la cantidad de marcas recordadas es mayor. Por último, la prueba de reconocimiento mide la capacidad de discriminar entre marcas previamente presentadas y otras alternativas plausibles. Es decir, es un continuo que va desde una recuperación autoguiada sin ningún tipo de ayuda o de organización hasta una tarea que simplemente implica discriminar información basándonos en la familiaridad (Richardson-Klavehn y Bjork, 1988; Tulving, 1985).

Cada prueba de memoria nos proporciona una información diferente, pero lo más habitual en los estudios de publicidad es centrarse en una o dos pruebas de memoria. Por ejemplo, Furham, Bergland y Gunter (2002) estudiaron el efecto que producía el hecho de que uno de los actores de una serie de televisión se bebiera una cerveza sobre el recuerdo de un anuncio de la misma bebida presentada en el corte publicitario anterior o posterior a esa escena. Los autores encontraron mejor memoria del contenido del anuncio con la prueba de recuerdo libre y de recuerdo con clave cuando la escena aparecía antes que el anuncio. También encontraron un efecto del orden de presentación del anuncio de cerveza dentro del corte publicitario, pero sólo en la tarea de recuerdo libre. Otros estudios se limitan a una sola prueba de memoria (Ferguson, Cruz, Martínez, Rueda y Ferguson, 2010; Martín-Luengo, Luna y Migueles, en prensa). Por ejemplo, Ferguson y colaboradores (2010) usaron una prueba de recuerdo libre para medir la memoria en función del tipo de anuncio, con contenido violento, sexual o neutro. Finalmente, otros estudios evaluaron la memoria con tareas de recuerdo libre, recuerdo con clave y reconocimiento pero agruparon los datos de las dos primeras pruebas en una única medida de memoria (Norris y Colman, 1996; Norris y cols., 2001; 2003).

Estudios previos apuntan a que con las tareas de recuerdo con clave se recupera una mayor cantidad de información respecto a la generada en las tareas de recuerdo libre (Tulving, 1985). Según el conocimiento de los autores no hay ningún estudio con publicidad sobre este tema, así que uno de nuestros objetivos es examinar las posibles diferencias entre ambas pruebas de memoria.Resultados previos han mostrado que hay una mayor recuperación de información con pruebas de memoria de recuerdo con clave que con recuerdo libre (Padilla-Walker y Poole, 2002; Paivio, Khan y Begg, 2000; Tulving, 1985). Siguiendo estos resultados, nuestra hipótesis es que en la prueba de recuerdo con clave se producirán una mayor cantidad de anuncios que en la prueba de recuerdo libre. Sin embargo, las características intrínsecas de la publicidad como la persuasión, repetición o intrusión hacen que la información que contiene pueda ser procesada de una manera diferente respecto de otro tipo de información, así que nuestra hipótesis es principalmente exploratoria.

También, se considera que la prueba de recuerdo libre es más difícil que la prueba de recuerdo con clave. En la prueba de recuerdo libre nuestro rendimiento depende únicamente de la información a la que somos capaces de acceder por nosotros mismos, lo que supone un esfuerzo cognitivo de búsqueda global, estrategia que hace que llegue un momento en el que somos incapaces de recuperar más información. Sin embargo, la prueba de recuerdo con clave facilita el acceso a nuestra memoria semántica (Tulving, 1985). Esto hace que el esfuerzo cognitivo que realizamos para recuperar los candidatos de una categoría sea menor debido a que se nos proporcionan las claves para acceder a la estructura de nuestro conocimiento (Algarabel, Pitarque y Dasi, 2002). Ante la presencia de una clave de recuperación, es probable que seamos capaces de recuperar más candidatos y alternativas. Por este motivo nuestra primera hipótesis es que en la prueba de recuerdo con clave los participantes producirán un mayor número de marcas que en la prueba de recuerdo libre.

Otro objetivo de este estudio es comprobar si el tipo de prueba de memoria determina la recuperación de marcas con diferentes valoraciones de interés. El interés se ha relacionado con el nivel de atención y también con el recuerdo de la información (Renniger y Wozniak, 1985). La información que consideramos interesante es capaz de atraer nuestro foco atencional, incluso cuando se presenta en la periferia de nuestro campo de visión, y es también la que mejor recordamos en una prueba de recuerdo libre respecto de otra información menos intere- 
sante (Renniger y Wozniak, 1985; Wade y Adams, 1990, Exp. 2). Dado que en una prueba de recuerdo libre recuperamos la información sin ningún tipo de guía externa, podemos esperar que los elementos recuperados sean aquellos a los que hemos prestado más atención y por lo tanto, aquéllos que consideramos más interesantes. Igualmente, en una prueba de recuerdo con clave el interés puede ser menor que en una prueba de recuerdo libre al estar indicada la categoría de lo que debemos recordar.

Por otro lado, hay que tener en cuenta que la cantidad de anuncios a los que estamos expuestos diariamente es enorme. En 2011 la inversión real estimada en el mercado publicitario español fue de 12.061 millones de euros, lo que supone el 1,12\% del PIB para ese mismo año según el informe INFOADEX (M.A. Sánchez, 2012). Esta sobreexposición a la publicidad, y el hecho de que algunas marcas se publiciten más que otras hace que estas sean más accesibles en nuestra memoria, que las consideremos más familiares e incluso se nos cree una cierta preferencia sobre otras marcas a las que estamos menos expuestos (Fransen, Fennis y Pruyn, 2010; Mantonakis, Bernstein y Loftus, 2011), aunque sean productos en los que no estamos interesados. Es decir, aunque a priori los anuncios sean de productos que no son de nuestro interés, podemos manifestar cierta preferencia por el hecho de haber sido expuestos a ellos de manera repetida. Ciertas marcas se vuelven accesibles e incluso las podemos llegar a recordar en una tarea de recuerdo con clave con valores altos en interés debido al efecto de la mera exposición (Zajonc, 1968). Este efecto implica que la simple exposición a un estímulo es condición suficiente para mejorar nuestras actitudes hacia él. Por lo tanto, aunque en principio en nuestro estudio esperaríamos un mayor nivel de interés en los elementos de la tarea de recuerdo libre, la sobreexposición a la publicidad de determinadas marcas y productos puede hacer que no encontremos diferencias en interés entre ambas pruebas.

En resumen, en la comparación de una prueba de recuerdo libre y otra de recuerdo con clave sobre marcas publicitarias esperamos un mayor número de marcas recuperadas en la tarea de recuerdo con clave, pero un mayor interés de las marcas recuperadas en la tarea de recuerdo libre. Para comprobar nuestras hipótesis pedimos a una muestra de participantes que completaran una prueba de recuerdo libre en la que tenían que listar el mayor numero de marcas/productos posibles y valorar el interés que les suscitaban. Seguidamente, los participantes completaron una prueba de recuerdo con clave en la que se les proporcionaban unas categorías de productos y debían indicar una marca y el interés por la misma. Finalmente, completaron unas preguntas generales sobre usos de los medios de comunicación en relación con la publicidad. Esta última batería de preguntas tiene como objetivo definir el perfil de la muestra en cuanto a los hábitos de los jóvenes en su interacción con los medios de información y los anuncios que contienen.

\begin{abstract}
Método

\section{Participantes}

Sesenta y cuatro alumnos (41 mujeres y 23 varones; edad media $=19,76$ años, $D T=4,79)$ de la Licenciatura de Psicología de la Universidad del País Vasco participaron voluntariamente en el experimento. Se han seguido las normas éticas pertinentes al tipo de procedimiento y población.
\end{abstract}

\section{Diseño}

Utilizamos un diseño de medidas repetidas de dos niveles: prueba de memoria (recuerdo libre, recuerdo con clave). Las medidas dependientes fueron la cantidad de marcas generadas y sus valoraciones de interés.

\section{Materiales}

Para realizar el estudio todos los participantes completaron dos pruebas de memoria sobre marcas de anuncios. También se presentaron varias preguntas sobre su interacción con la publicidad en diversos medios como la radio, prensa y televisión.

Prueba de recuerdo libre. En la primera prueba se pidió a los participantes que enumerasen los anuncios o marcas de productos que recordaran, que indicaran el medio al que pertenecían y el nivel de interés en una escala de 1 a 6 , siendo 1 nada interesante y 6 muy interesante. Para completar este cuestionario disponían de 5 minutos.

Prueba de recuerdo con clave. La segunda prueba constaba de 27 categorías de productos (ver Anexo 1) para los cuáles debían indicar una marca o anuncio y el interés de 1 a 6. Si no recordaban el nombre de ninguna marca de alguna de las categorías, debían indicar su interés en productos de esa categoría en general. Un total de 13 de las categorías proporcionadas se extrajeron del estudio de Jóvenes y Publicidad (L. Sánchez y cols., 2004). Se añadieron 14 categorías generadas por los autores de productos para los cuáles los jóvenes no son el público objetivo. Estas 14 categorías se incluyeron para evitar que las valoraciones de interés fueran realizadas exclusivamente sobre productos diseñados para jóvenes, lo que podría aumentar artificialmente las valoraciones. El objetivo fue tener un rango amplio de productos que pudiera ser comparable con la completa falta de limitaciones de la prueba de recuerdo libre.

Preguntas generales. Por último, los participantes completaron unas preguntas genéricas sobre anuncios en los medios de radio, televisión y prensa (ver Anexo 2).

\section{Procedimiento}

El experimento se llevó a cabo en grupos reducidos tras una práctica de aula en la que se solicitaron voluntarios para hacer el experimento. Se repartieron los cuadernillos con las tres pruebas de memoria. Se informó a los participantes que no volvieran la hoja hasta que se les avisara y se explicó cómo realizar la primera prueba de memoria. Todos participantes hicieron las pruebas en el mismo orden: primero la tarea de recuerdo 
libre, en segundo lugar el reconocimiento, y por último las preguntas generales. No hubo contrabalanceo de pruebas para evitar sesgos en la producción. Si los participantes dispusieran en primer lugar de las categorías de los productos (recuerdo con clave), tenderían a reproducirlas en la tarea de recuerdo libre. Es decir, se podría dar un efecto de anclaje hacia los productos mostrados en la tarea de recuerdo con clave. Para completar la primera prueba de recuerdo libre tuvieron un total de cinco minutos en los que debían intentar recordar activamente marcas de productos y realizar la valoración de interés sobre las mismas. Tras ese tiempo se les dijo que podían volver la página del cuadernillo, se explicó la segunda tarea, se informó que no tenían límite de tiempo y que una vez hubieran finalizado debían contestar las preguntas de la última hoja. Cuando todos los participantes terminaron de completar los cuadernillos se explicaron los objetivos del experimento y se respondieron las preguntas surgidas de las tareas.

\section{Resultados \\ Cantidad de marcas recuperadas}

Se realizó un análisis de varianza (AVAR) de medidas repetidas sobre la cantidad de marcas recuperadas en ambas pruebas. En la prueba de recuerdo con clave los participantes recordaron más marcas $(M=11,37, D T=7,80)$ que en la prueba de recuerdo libre $(M=5,89, D T=2,60), F(1,63)=29,61, M S E$ $=973,51, p<.001, \eta_{p}{ }^{2}=.32$. Este resultado viene a apoyar la hipótesis de que con una tarea de recuerdo con clave se recupera una mayor cantidad de información, en este caso marcas, que en una de recuerdo libre.

\section{Interés de las marcas}

De la tarea de recuerdo con clave se obtuvieron valores de interés asociados a una marca específica y, cuando no se recuperó ninguna marca, valores de interés asociados a la categoría. Para estudiar si se dan diferencias en los valores de interés entre ambas variables se realizó un AVAR de medidas repetidas. Encontramos valores de interés superiores cuando los participantes fueron capaces de nombrar una marca para los productos solicitados $(M=3,01, D T=0,99)$ que cuando han sido incapaces de nombrar una marca concreta $(M=2,53, D T=.89)$, $F(1,52)=31,28, M S E=8,17, p<.001, \eta p 2=.38$. Como hubo diferencias en las evaluaciones de interés entre ambos tipos de información, para la comparación con la prueba de recuerdo libre sólo se tuvieron en cuenta los datos de interés cuando se ha dado el nombre de una marca.

Para analizar las diferencias de las evaluaciones de interés entre las pruebas de recuerdo libre y con clave se realizó un AVAR de medidas repetidas con las proporciones de las evaluaciones de interés de ambas pruebas. Se encontró que los valores de interés en la prueba de recuerdo libre $(M=5,86, D T=2,60)$ fueron mayores que en la prueba de recuerdo con clave $(M=$ $3,11, D T=0,99), F(1,63)=54,99, M S E=240,52, p<.001, \eta_{p}{ }^{2}$ $=.466$. Este resultado apoya nuestra segunda hipótesis de un mayor nivel de interés para las marcas generadas sin ayuda de claves de recuperación.

\section{Preguntas generales sobre los medios y la publicidad}

En relación a las respuestas sobre la radio y su publicidad, el $35 \%$ de la muestra dijo que escuchaban asiduamente la radio durante una media al día de 85,63 minutos. Todos los participantes dijeron escuchar programas musicales, y además, el $9 \%$ también escuchan programas informativos, el 5\% programas despertador, y un $1 \%$ programas nocturnos, tertulia o entretenimiento. Un 33\%, de los participantes que escuchan la radio respondieron que los programas contenían anuncios. El 18\% dice que no cambia de emisora mientras se emiten los anuncios y el $16 \%$ que sí, de los cuales el $7 \%$ lo hacen porque son aburridos.

Respecto a las respuestas recogidas en relación a la televisión, del total de la muestra el $83 \%$ afirmaron ver asiduamente la televisión una media de 116,09 minutos al día. El tipo de programas que ven se reparten en: entrenamiento $28 \%$, series el $28 \%$, informativos $12 \%$, películas un $6 \%$, dibujos animados $5 \%$, música $3 \%$ y una persona afirmó ver programas de cultura/ tertulia/documental e historia. El 64\% indicó que los programas que ven tienen anuncios y un 58\% de ellos afirmaron cambiar de canal. La mayoría dicen que cambian por aburrimiento, porque suelen ser muchos, para poder ver varios programas a la vez, o por no verlos.

Finalmente, en relación a las preguntas sobre los medios en papel, un $51 \%$ de la muestra afirmó leer algún tipo de prensa. A ello dedican una media diaria de lectura de 33,79 minutos. Un $20 \%$ leen periódicos, un $9 \%$ leen revistas, de las cuáles indican que son de cotilleo $5 \%$, de moda el $2 \%$, prensa deportiva un $1 \%$ y sobre informática también un $1 \%$. El $8 \%$ de la muestra respondió que leen varios tipos de prensa sin especificar. El 42 $\%$ de los participantes dicen que la prensa que leen contiene anuncios, mientras que 3\% dicen que leen prensa sin anuncios publicitarios. Entre los que afirman leer prensa que contiene anuncios, un $14 \%$ dice leerlos y el $27 \%$ no. Entre las razones que dan los participantes que leen los anuncios están porque les resultan bonitos, son de trabajo, de moda, es inevitable ya que están en medio de la información y se te va la vista, son divertidos e interesantes o porque están relacionados con el tema que trata el artículo de la revista. Entre el $27 \%$ que dicen esquivarlos la mayoría argumenta que lo hace porque son aburridos o porque no les interesan.

\section{Discusión}

Los dos objetivos principales de este trabajo han sido estudiar la influencia del tipo de prueba de memoria en la cantidad de marcas recuperadas y en las evaluaciones de interés de las mismas. Los resultados obtenidos apoyan la hipótesis de un mayor número de marcas recuperadas con una prueba de recuerdo con clave, y de mayores valores de interés de las marcas recuperadas en la prueba de recuerdo libre. 
Tal como esperado, los resultados muestran que en la prueba de recuerdo con clave se recupera un número mayor de marcas que en la prueba de recuerdo libre. Este resultado puede explicarse debido a que una prueba en la que se nos facilitan pistas sobre el tipo de información por la que nos están preguntando siempre va a ser más fácil respecto de otra en la que tenemos que recuperar información sin ningún tipo de clave (Till y Baack, 2005). La recuperación de la información en una tarea de memoria está determinada en parte por su accesibilidad en ese momento, y en principio lo esperable es que la accesibilidad sea mayor en la tarea de recuerdo con clave respecto de una de recuerdo libre (Schwarz y Salzberg, 1975). El recuerdo libre requiere que el participante espontáneamente utilice estrategias de búsqueda eficaces, tarea que requiere mayor esfuerzo cognitivo y elaboración que una prueba dirigida por claves específicas. Sin embargo, parte de la cantidad de marcas recuperadas puede deberse al orden en el que se realizaron las pruebas de memoria. Varios autores indican que la realización de una prueba de recuerdo libre anterior a otra prueba de memoria puede aumentar el rendimiento en la segunda (Brewer, Marsh, Meek, Clark-Foos y Hicks, 2010; Chan y McDermott, 2007). No obstante, la diferencia de producción entre ambas pruebas es tan alta que probablemente con un diseño de medidas independientes también hubiéramos encontrado diferencias significativas en el mismo sentido.

Respecto a las valoraciones de interés, las marcas que somos capaces de producir por nosotros mismos son valoradas como más interesantes que cuando hemos tenido claves externas que ayudan a producirlas. Una posible explicación para este resultado es que las marcas que recordamos en una y otra prueba hayan sido procesadas a diferente nivel. La memoria de un ítem, en este caso un anuncio, está relacionada con la intensidad del procesamiento del mismo (Craik y Tulving, 1975). Un procesamiento profundo implica una mayor elaboración del significado de la información respecto de un procesamiento superficial en el que apenas se retienen aspectos generales de la misma. Un procesamiento profundo se ha relacionado con una mejor memoria (Mandler, 1967). Así, podríamos suponer que somos capaces de producir anuncios sin claves externas cuando hemos realizado un procesamiento profundo de los mismos. Los anuncios interesantes podrían llamar nuestra atención y ser procesados de manera más profunda, y por eso ser susceptibles de ser recuperados sin ningún tipo de clave externa. En apoyo a estas ideas, Shavitt, Vargas y Lowrey (2004) encontraron una memoria más completa para los anuncios para los que los participantes mencionaron que habían prestado atención.

En nuestro estudio también nos interesamos en caracterizar la muestra de participantes en función de su interacción con los medios y la publicidad a través de sus respuestas en las preguntas generales finales. Este aspecto nos parece importante ya que ayuda a la interpretación y generalización de los resultados obtenidos. Por ejemplo, es interesante señalar que un gran número de nuestros participantes esquivan los anuncios, prin- cipalmente porque son aburridos. Esto puede explicar la tendencia a cambiar de canal cuando empieza la tanda de anuncios en un corte publicitario. Es posible que si los anuncios fueran más creativos se consiguiera que espectadores semejantes a los que han participado en esta investigación no cambiaran de canal (Till y Baack, 2005). También es importante caracterizar la muestra para facilitar la comparación con futuras investigaciones. Por ejemplo, los resultados podrían ser distintos si este experimento fuera replicado con una muestra diferente, como personas mayores con diferentes intereses y usos de los medios de comunicación que los jóvenes que han participado en este experimento, o expertos en publicidad con un gran interés en los anuncios. Los datos reportados de uso de medios y reacciones ante los anuncios pueden ayudar a explicar esas diferencias si existieran.

Como conclusión, dependiendo del objetivo que tengamos tendremos que utilizar una prueba de memoria u otra. Así, si lo que buscamos es conocer la mayor cantidad de marcas que conoce un tipo de consumidor determinado, deberemos usar una prueba de memoria con clave. Si, por el contrario, lo que nos interesa es saber las marcas que son más interesantes para ese mismo tipo de consumidor, la prueba de recuerdo libre es más adecuada. En cualquier caso, a la hora de tomar una medida de memoria debemos tener en cuenta que dependiendo de qué tipo de prueba utilicemos la información obtenida será diferente. Las medidas de memoria son el indicador más utilizado en las campañas de marketing para medir el impacto de los anuncios, por lo que la elección de la prueba de memoria adecuada puede ser el detalle que haga la diferencia.

\section{Referencias}

1. Algarabel, S., Pitarque, A. y Dasí, C. (2002). Efecto del tipo de información sobre el esfuerzo cognitivo y el estado de consciencia asociado al recuerdo. Psicothema, 14, 303-308.

2. Brewer, G. A., Marsh, R. L., Meek, J. T., Clark-Foos, A. y Hicks, J. L. (2010). The effects of free recall testing on subsequent source memory. Memory, 18, 385-393. http:// dx.doi.org/10.1080/09658211003702163

3. Chan, J. C. K. y McDermott, K. B. (2007). The testing effect in recognition memory: A dual process account. Journal of Experimental Psychology: Learning, Memory, and Cognition, 33, 431-437. http://dx.doi.org/10.1037/02787393.33.2.431

4. Craik, F. I. y Tulving, E. (1975). Depth of processing and the retention of words in episodic memory. Journal of Experimental Psychology: General, 104, 268-294. http:// dx.doi.org/10.1037/0096-3445.104.3.268

5. Fennis, B. M. y Stroebe, W. (2010). The psychology of advertising. Hove, East Sussex: Psychology Press.

6. Ferguson, C. J., Cruz, A. M., Martínez, D. Rueda, A. y Ferguson, D. E. (2010). Violence and sex advertising strategies in television commercials. European Psychologist, 15, 304311. http://dx.doi.org/10.1027/1016-9040/a000016 
7. Fransen, M. L., Fennis, B. M. y Pruyn, A. Th. H. (2010). Matching communication modalities: The effects of modality congruence and processing style on brand evaluation and brand choice. Communication Research, 37, 576598.

8. Furham, A., Bergland, J. y Gunter, B. (2002). Memory for television advertisements as a function of advertisementprogramme congruity. Applied Cognitive Psychology, 16, 525-545. http://dx.doi.org/10.1002/acp.812

9. Goldsmith, M., Koriat, A. y Weinberg-Eliezer, A. (2002). Strategic regulation of grain size in memory reporting. Journal of Experimental Psychology: General, 131, 73-95. http://dx.doi.org/10.1037//0096-3445.131.1.73

10. Luna, K., Higham, P. A. y Martín-Luengo, B. (2011). Regulation of memory accuracy with multiple answers: the plurality option. Journal of Experimental Psychology: Applied, 17, 148-158. http://dx.doi.org/10.1037/a0023276

11. Mandler, G. (1967). Organization and memory. En K. W. Spence y J. T. Spence (Eds.), The psychology of learning and motivation: Advances in research and theory (pp. 328372). New York: Academic Press.

12. Mantonakis, A., Bernstein, D. M. y Loftus, E. F. (2011). Attributions of fluency: Familiarity, preference, and the senses. En P. A. Higham y J. P. Leboe (Eds.), Constructions of remembering and metacognition. Essays in honour of Bruce Whittlesea (pp. 40-50.). Basingstoke, Reino Unido: Palgrave Macmillan.

13. Martín-Luengo, B., Luna, K. y Migueles, M. (en prensa). Memory for radio advertisements: effects of programme and typicality. The Spanish Journal of Psychology.

14. Norris, C. E. y Colman, A. M. (1996). Context effects of radio programming on cognitive processing of embedded advertisements. Applied Cognitive Psychology, 10, 473-486. http://dx.doi.org/10.1002/(SICI)10990720(199612)10:6<473::AID-ACP390>3.0.CO;2-\#

15. Norris, C. E., Colman, A. M. y Aleixo, P. A. (2001). Context effects of cognitively involving, entertaining and enjoyable television programmes on two types of advertisements. Social Psychological Review, 3, 3-24.

16. Norris, C. E., Colman, A. M. y Aleixo, P. A. (2003). Selective exposure to television programmes and advertising effectiveness. Applied Cognitive Psychology, 17, 593-606. http://dx.doi.org/10.1002/acp.892

17. Padilla-Walker, L. M. y Poole, D. A. (2002). Memory for previous recall: a comparison of free and cued recall. Applied Cognitive Psychology, 16, 515-524. http://dx.doi. org/10.1002/acp.809

18. Paivio, A., Khan, M. y Begg, I. (2000). Concreteness and relational effects on recall of adjective-noun pairs. Canadian Journal of Experimental Psychology, 54, 149-159. http://dx.doi.org/10.1037/h0087337

19. Renniger, K. A. y Wozniak, R. H. (1985). Effect of interest on attentional shift, recognition, ad recall in young children. Developmental Psychology, 21, 624-632. http:// dx.doi.org/10.1037/0012-1649.21.4.624

20. Richardson-Klavehn, A. y Bjork, R. A. (1988). Measures of memory. Annual Review of Psychology, 39, 475-543. http:// dx.doi.org/10.1146/annurev.ps.39.020188.002355

21. Sánchez, M. A. (2012). Resumen del estudio INFOADEX de la inversión publicitaria en España 2012. Madrid: INFOADEX, S. A.

22. Sánchez, L., Megías, I. y Rodríguez, E. (2004). Jóvenes y Publicidad. Valores en la comunicación publicitaria para jóvenes. Instituto de la Juventud de España (INJUVE). Madrid: Fundación de Ayuda contra la Drogadicción (FAD) y Caja Madrid.

23. Schwarz, W. y Salzberg, P. M. (1975). Free-recall performance as a function of input cue accessibility. Journal of Experimental Psychology: Human, Learning, and Memory, 1, 746-771. http://dx.doi.org/10.1037/0278-7393.1.6.764

24. Shavitt, S. Vargas, P. y Lowrey, P. (2004). Exploring the role of memory for self-selected ad experience: Are some advertising media better liked than others? Psychology \& Marketing, 21, 1011-1032. http://dx.doi.org/10.1002/ $\underline{\text { mar.20035 }}$

25. Till, B. D. y Baack, D. W. (2005). Recall and persuasion: Does creative advertising matter? Journal of Advertising, 34, 47-57.

26. Tulving, E. (1985). Memory and consciousness. Canadian Psychologist, 26, 1-12. http://dx.doi.org/10.1037/h0080017

27. Wade, S. E. y Adams, R. B. (1990). Effects of importance and interest on recall of biographical text. Journal of Reading Behaviour, 4, 331-353. http://dx.doi. org/10.1080/10862969009547717

28. Zajonc, R. B. (1968). Attitudinal effects of mere exposure. Journal of Personality and Social Psychology , 9, 1-27. http://dx.doi.org/10.1037/h0025848

Fecha de recepción: 29 de junio de 2012 Fecha de recepción de la versión modificada: 9 de octubre de 2012 Fecha de aceptación: 6 de noviembre de 2012 


\section{PRUEBA DE MEMORIA E INTERÉS}

Anexo 1

Categorías de productos utilizadas en la tarea de recuerdo con clave. Las categorías marcadas con un asterisco son las tomadas del estudio de Sánchez, Megías y Rodríguez (2004).

$\begin{array}{lll}\text { *Ropa } & \text { *Ordenador } & * \text { Autoescuela } \\ \text { *Librería } & \text { Belleza } & \text { Limpieza hogar } \\ * \text { Colonias } & \text { *Electrónica } & \text { Electrodomésticos } \\ * \text { *ámara fotos } & \text { *Idiomas } & \text { Banco/pensiones } \\ * \text { Móvil } & \text { Muebles } & \text { Cuidado animal } \\ * \text { *oches } & \text { Restaurante } & \text { Seguro coche } \\ * \text { Mp3 } & \text { Clínica dental } & \text { Inmobiliaria } \\ \text { Viajes } & \text { Instrumentos musicales } & \text { Fontanería } \\ * \text { Ropa deportiva } & * \text { Gimnasio } & \text { Mudanza }\end{array}$

\section{Anexo 2}

Preguntas sobre la interacción con la publicidad de los medios.

Anuncios de RADIO

¿Sueles escuchar la radio? SI NO ¿Cuántas horas al día?

¿Qué tipo de programas?

¿Tienen anuncios? SI NO ¿Cambias de emisora durante los anuncios? SI NO

Si lo haces, ¿por qué?

Anuncios de TV

¿Sueles ver la TV? SI NO ¿Cuántas horas al día?

¿Qué tipo de programas?

¿Tienen anuncios? SI NO ¿Cambias de canal durante los anuncios? SI NO

Si lo haces, ¿por qué?

Anuncios de PRENSA/REVISTAS/PERIÓDICOS:

¿Sueles leer prensa escrita? SI NO ¿Cuánto tiempo dedicas al día? ¿Qué tipo de prensa?

¿Tienen anuncios? SI NO ¿Lees los anuncios? SI NO

Si lo haces, ¿por qué? 